\title{
High-Intensity Laser Triggered Proton Acceleration from Ultrathin Foils
}

\author{
A. Brantov ${ }^{1 *}$, V. Yu. Bychenkov ${ }^{1}$, D. V. Romanov ${ }^{2}$, F. Dollar ${ }^{3}$, A. Maksimchuk ${ }^{3}$, \\ and K. Krushelnick ${ }^{3}$ \\ ${ }^{1}$ P. N. Lebedev Physics Institute, Russian Academy of Sciences, Moscow 119991, Russia \\ 2 All-Russia Research Institute of Automatics, Moscow 127055, Russia \\ ${ }^{3}$ Center for Ultrafast Optical Science, University of Michigan, Ann Arbor, Michigan 48109, USA
}

Received 28 May 2012, accepted 29 October 2012

Published online 01 February 2013

Key words Laser triggered proton acceleration, particle-in-cell, Thomson Parabola, preplasma, ultrathin foil, tight focusing.

The recently developed PIC code MANDOR features arbitrary target design including 3D preplasma and the 6-component laser fields of a tightly focused laser beam. The 3D simulations have been performed to model recent HERCULES experiments on proton acceleration, where protons with energy greater than $20 \mathrm{MeV}$ were produced using just $1.5 \mathrm{~J}$ laser pulses focused to intensity of $2 \times 10^{21} \mathrm{~W} / \mathrm{cm}^{2}$. By adapting the 3D target geometry relating to ps-prepulse effect, reasonable agreement with experimental data for the proton energy spectrum has been achieved. The effect of the 3D preplasma shape on efficiency of proton acceleration is discussed.

Laser ion acceleration is an attractive field of research due to the possible applications of ion beams for laser fusion, modeling of astrophysical phenomena in a laboratory, nuclear physics, radiography, and medicine [1]. Modern laser systems are able to produce femtosecond, multi-terawatt laser pulses which can be tightly focused to a focal spot with dimensions on the order of the laser wavelength, resulting in $10^{21}-10^{22} \mathrm{~W} / \mathrm{cm}^{2}$ intensities. The interaction of such intense laser pulses with ultra-thin targets is expected to advance ion beam generation in terms of the maximum ion energy. The PIC simulations predict ion energy at the level of $100 \mathrm{MeV} /$ nucleon generated from the ideal target with an optimal thickness and with a sharp boundary for $\sim 10 \mathrm{~J}$ of laser pulse [2], although that has never been demonstrated in the experiments to date. At the same time even a small laser pre-pulse is able to significantly modify the ideal target geometry. In spite of the possibility to produce almost ideal laser pulse contrast $\left(10^{-13}\right)$ at the nano-second time scale, the laser pulse is still far from Gaussian at the picosecond and even $\sim 10$ ps time scale. This can produce a 3D-preplasma before the peak of the laser pulse arrives. Such preplasma formation on even submicron scale lengths may strongly affect the spectra of the accelerated ions.

Let us consider the interaction of a linearly polarized ultrashort (30 fsec) laser pulse with a peak dimensionless laser field amplitude of $a_{0}=30$ (corresponding to an intensity of $2 \times 10^{21} \mathrm{~W} / \mathrm{cm}^{2}$ for a laser wavelength of $0.8 \mu \mathrm{m})$ with a thin, fully ionized target consisting of carbon ions $\left(C^{6+}\right)$, protons and electrons. The laser pulse is tightly focused with an off-axis parabolic mirror with $f_{\#}=1$. The target has an electron density of $200 n_{c r}$, which corresponds to a real mass density of $\mathrm{CH}\left(1.1 \mathrm{~g} / \mathrm{cm}^{3}\right)$. For this laser pulse intensity and target density, the optimum target thickness should be about $0.05 \lambda\left(l_{0} \sim \lambda a_{0} n_{c} / \pi n_{e}\right)$ [3,4]. This result matched nicely by our 3D simulations of proton acceleration from the foil with an ideal target-vacuum interface. We found that the optimum $\mathrm{CH}$ target thickness is on the order of $50 \mathrm{~nm}$ (see. Fig. 1). We believe that such thin targets may be significantly affected by the picosecond laser contrast.

Along with a plain foil with a sharp boundary and with thickness of $100 \mathrm{~nm}$ (a), we also used modified targets with a small preplasma inside the focal spot (as transversal size) having an exponential density profile (with a characteristic scale of $100 \mathrm{~nm}$ ) either at the front side (b) or symmetrically at both sides, front and back of the foil (c) (see. Fig. 2). The density profile of an expanded plasma in transverse directions in cases (b) and

* Corresponding author. E-mail: brantov@lebedev.ru 
(c) was a Gaussian with characteristic scale of $1.5 \mu \mathrm{m}$, corresponding to the laser spot size. To compare with experimental data (see below) for $100 \mathrm{~nm}$ foils, we accounted for nonideal several-ps-slope of the laser pulse which may result in target expansion up to $100 \mathrm{~nm}$ during $\sim$ several $(10 \mathrm{ps})$ picoseconds with $\sim 10^{13} \mathrm{~W} / \mathrm{cm}^{2}$ prepulse intensity (see, for example [5]). The spectra of protons accelerated from these targets are also shown in Fig. 2. The small-size preplasma formed from the target expansion in the backward direction has almost no effect on proton spectrum. There is some small redistribution of energetic protons near maximum energy cut-off. The plasma expansion in both directions results in decrease of proton maximum energy, which is due to acceleration field decrease because of destruction of sharp rear target boundary.

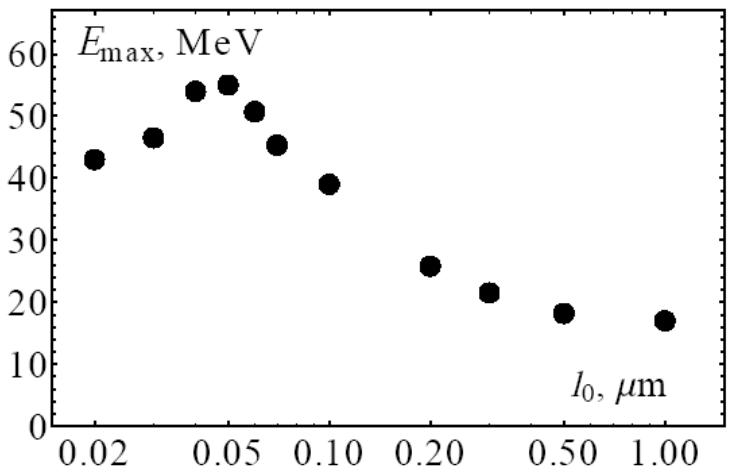

Fig. 1 Maximum proton energy $(\mathrm{MeV})$ versus target thickness $(\mu \mathrm{m})$ elaborated from $3 \mathrm{D}$ simulations.

Significant differences in these aforementioned cases appear in the proton energy spectra, propagated in an opening angle of $1^{\circ}$ with respect to the direction of target normal (Fig. 3). The plasma expansion in both direction results in an increase of protons with energy in the interval of $10-15 \mathrm{MeV}$ and significant decrease of energetic protons with energy greater $20 \mathrm{MeV}$. In this case spectrum has a thermal-like decreasing shape with cut-off at $\sim 30 \mathrm{MeV}$ and some gap near $7 \mathrm{MeV}$, which is relevant to light and heavy ion plasma expansion in agreement with the theory [6]. The proton spectrum from an ideal plain foil is flat for the high-energy protons. The maximum number of hot protons almost constant in the range $10 \mathrm{MeV}-35 \mathrm{MeV}$.

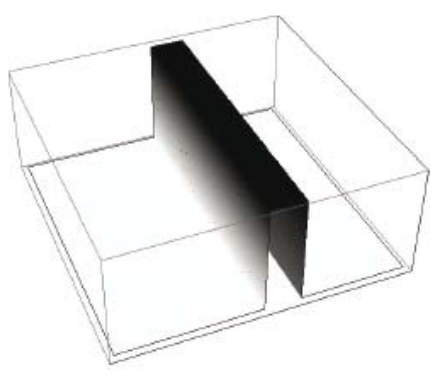

a)

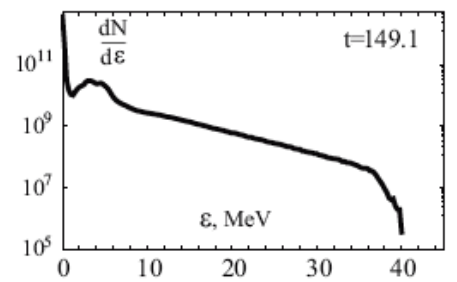

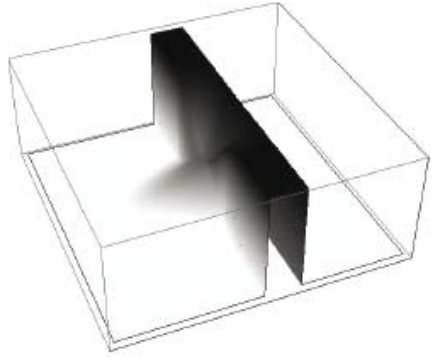

b)

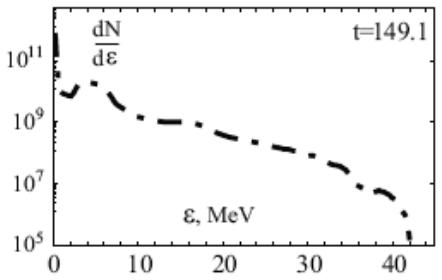

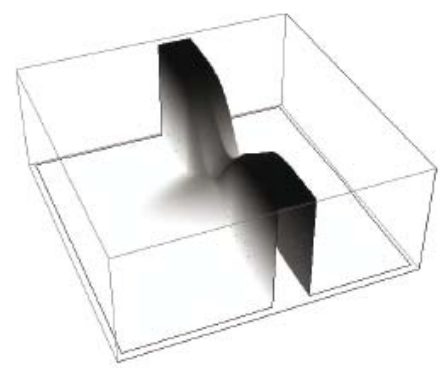

c)

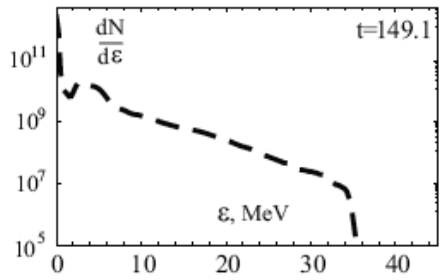

Fig. 2 Sketch of target density profiles (in X-Y plane, $\mathrm{X}$ is direction of laser beam propagation) and corresponding spectra of the protons (number of protons per $\mathrm{MeV}$ ) for the targets with different preplasma at time of $149.1 \mathrm{fs}$. Solid curve corresponds to plain foil (case a), dash-dots curve (case b) and dashed curve (case c) correspond to the targets expanded in the front side or symmetrically in both sides.

The experiments were performed using the HERCULES laser facility at University of Michigan, a Ti:Sapphire system $(\lambda=800 \mathrm{~nm})$ producing laser pulses with $\tau=30 \mathrm{fs}$ duration full width at half maximum (FWHM) and an 
amplified spontaneous emission intensity contrast of $10^{-11}$. With implementation of two plasma mirrors the nanosecond laser contrast was further improved by 5 orders of magnitude and the picosecond $(\sim 10$ ps $)$ contrast became as high as $\sim 10^{-8}-10^{-9}$ [7]. In these experiments, the laser delivered $\sim 1.5 \mathrm{~J}$ to the target with up to $70 \%$ of the energy in a $1.2 \mu \mathrm{m}$ FWHM focal spot via an $f_{\#}=1$ off-axis parabolic mirror. This results in an on-target intensity of $2 \times 10^{21} \mathrm{~W} / \mathrm{cm}^{2}$ (corresponding to $a_{0}=30$ ). The targets used in the experiment were freestanding $100 \mathrm{~nm}$ polypropylene $\left(\left(C_{3} H_{6}\right)_{n}\right)$ foils. The targets were positioned at the laser focus with an accuracy of $2 \mu \mathrm{m}$ at normal incidence. The experiments were performed with linearly polarized light. A Thomson parabola ion spectrometer in the rear target normal direction was the primary diagnostics for the measurements of ion energy spectra and the maximum ion energy and the total proton numbers at high energies were simultaneously measured using CR-39 track detectors with steps of different thicknesses filters so that the minimum stopping distance for each bin could be calculated using SRIM [8]. The highest proton energy observed in these experiments were $21 \mathrm{MeV}$ as observed on the Thomson Parabola, and is confirmed via the CR-39 detector as seen in Fig. 4.

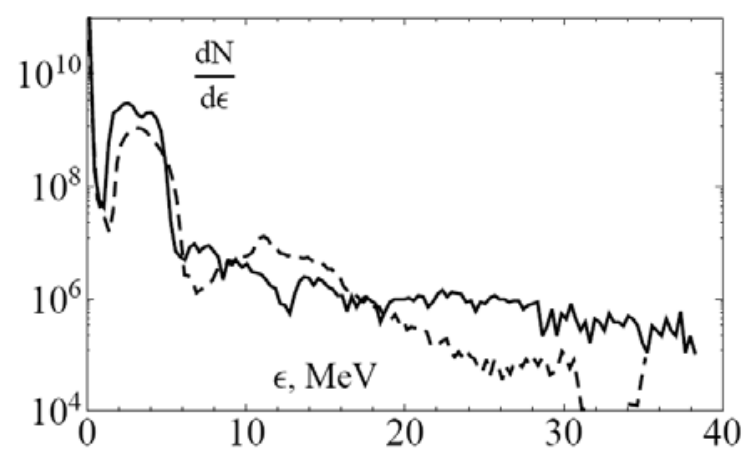

Fig. 3 Spectra of protons (number of protons per MeV), propagated in the opening angle of $1^{\circ}$. Solid line corresponds to plain foil (case a) and dashed line corresponds to target with plasma expanded at both directions (case c).

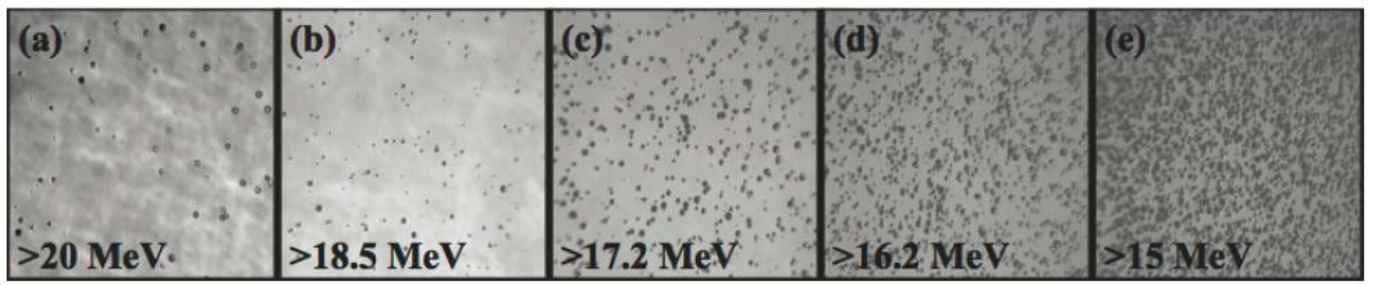

Fig. 4 CR39 tracks for proton hits for a variety of energy bins. Minimum stopping power for each bin is $20 \mathrm{MeV}$ (a), 18.5 $\mathrm{MeV}$ (b), 17.2 MeV (c), 16.2 MeV (d), and $15 \mathrm{MeV}$ (e) as determined by SRIM calculations.

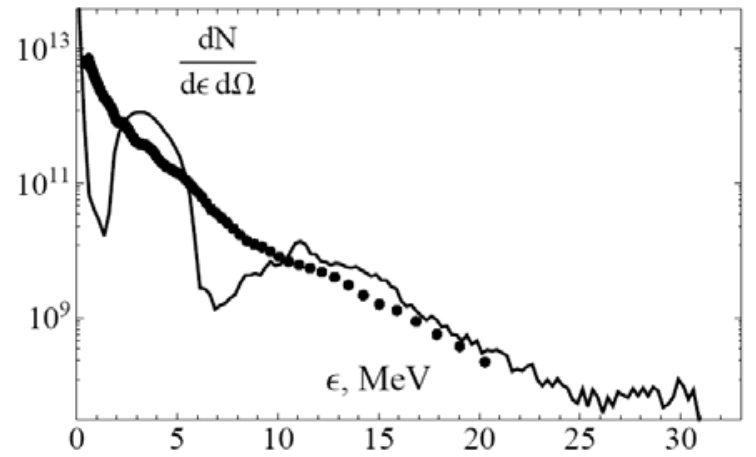

Fig. 5 Experimental (dots) and calculated (solid line for case c) spectra of the accelerated protons (number of protons per $\mathrm{MeV}$ per Steradian).

The comparison of the experimental and theoretical spectra is presented in Fig. 5. The calculated spectrum for case (c) has the same slope as the energetic protons with energy greater than $10 \mathrm{MeV}$. Appearing of rather deep gap in proton spectra in the simulation could be due to ignoring multi-species carbon ions $\left(C^{+6}, C^{+5}, C^{+4} \ldots\right)$ and some underestimation of hydrogen concentration in a target $\left(\mathrm{CH}\right.$ instead of $\left.\mathrm{CH}_{2}\right)$. This strongly suggests 
that for the case of submicron foils, the expansion from both rear and front surfaces does occur due to preplasma formation on the picosecond timescale. This also may explain the lower maximum proton energies observed experimentally for thin foils compared to the result of simulations without the preplasma, indicating that further increases in laser contrast may enhance the proton spectra produced.

This work is supported by the Russian Foundation for Basic Research (grants 10-02-91060-NCNIa, 10-0200925-a, 12-02-00231-a, 12-02-01161-a, 12-02-33045-mol_a_ved), the Ministry of education and science of Russian Federation, project \# 2012-1.2.2-12-000-1011-055, and the Office of Naval Research Laboratory (ONRL).

\section{References}

[1] M. Borghesi, J. Fuchs, S. V. Bulanov et al., Fus. Sci. and Tech. 49, 412 (2006).

[2] A.V. Brantov, V.Yu. Bychenkov, K.I. Popov et al., Nucl. Instr. and Meth. A 653, 62 (2011).

[3] A.V. Brantov, V.Yu. Bychenkov, and W. Rozmus, Quantum Electronics 37, 863 (2007).

[4] T.Z. Esirkepov, S.V. Bulanov, K. Nishihara et al., Phys. Rev. Lett. 89, 1753003 (2002).

[5] A.G. Mordovanakis, J. Easter, N. Naumova et al., Phys. Rev. Lett. 103, 235001 (2009).

[6] V.T. Tikhonchuk, A.A. Andreev, S.G. Bochkarev, and V. Yu. Bychenkov, Plasma Phys. Control. Fusion B 47, 869 (2005).

[7] F. Dollar, C. Zulick, A.G.R. Thomas et. al., Phys. Rev. Lett. 108, 175005 (2012).

[8] J. F. Ziegler, Nucl. Instr. and Meth. B 219, 1027 (2004). 